\title{
Lepidopteran HMG-CoA reductase is a potential selective target for pest control
}

\author{
Yuan-mei Li ${ }^{1}$ ， Zhen-peng Kai ${ }^{\text {Corresp.. }}{ }^{1}$, Juan Huang ${ }^{2}$, Stephen S Tobe ${ }^{2}$ \\ ${ }^{1}$ School of Chemical and Environmental Engineering, Shanghai Institute of Technology, Shanghai, China \\ 2 Department of Cell and Systems Biology, University of Toronto, Toronto, Ontario, Canada \\ Corresponding Author: Zhen-peng Kai \\ Email address: kaizp@sit.edu.cn
}

As a consequence of the negative impacts on the environment of some insecticides, discovery of eco-friendly insecticides and target has received global attention in recent years. Sequence alignment and structural comparison of the rate-limiting enzyme HMGCoA reductase (HMGR) revealed differences between lepidopteran pests and other organisms, which suggested insect HMGR could be a selective insecticide target candidate. Inhibition of JH biosynthesis in vitro confirmed that HMGR inhibitors showed a potent lethal effect on the lepidopteran pest Manduca sexta, whereas there was little effect on JH biosynthesis in Apis mellifera and Diploptera punctata. The pest control application of these inhibitors demonstrated that they can be insecticide candidates with potent ovicidal activity, larvicidal activity and insect growth regulatory effects. The present study has validated that Lepidopteran HMGR can be a potent selective insecticide target, and the HMGR inhibitors (especially type II statins) could be selective insecticide candidates and lead compounds. Furthermore, we demonstrated that sequence alignment, homology modeling and structural comparison may be useful for determining potential enzymes or receptors which can be eco-friendly pesticide targets. 
1

2

3

4

5

6 Shanghai, 201418, P.R. China

7 $8 \quad$ Canada M5S $3 G 5$

9

10

11

$12 *$ Corresponding author

13 Email: kaizp@sit.edu.cn

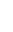

10

\section{target for pest control \\ Lepidopteran HMG-CoA reductase is a potential selective}

Yuan-mei Li ${ }^{1}$, Zhen-peng Kai ${ }^{1, *}$, Juan Huang ${ }^{2}$, Stephen S Tobe ${ }^{2}$

${ }^{2}$ Department of Cell and Systems Biology, University of Toronto, 25 Harbord St., Toronto, Ont.,

14

15

16

17

18

19 


\section{Abstract}

As a consequence of the negative impacts on the environment of some insecticides,

23 discovery of eco-friendly insecticides and target has received global attention in recent years.

24 Sequence alignment and structural comparison of the rate-limiting enzyme HMG-CoA

25 reductase (HMGR) revealed differences between lepidopteran pests and other organisms,

26 which suggested insect HMGR could be a selective insecticide target candidate. Inhibition of

27 JH biosynthesis in vitro confirmed that HMGR inhibitors showed a potent lethal effect on the

28 lepidopteran pest Manduca sexta, whereas there was little effect on JH biosynthesis in Apis

29 mellifera and Diploptera punctata. The pest control application of these inhibitors

30 demonstrated that they can be insecticide candidates with potent ovicidal activity, larvicidal

31 activity and insect growth regulatory effects. The present study has validated that

32 Lepidopteran HMGR can be a potent selective insecticide target, and the HMGR inhibitors

33 (especially type II statins) could be selective insecticide candidates and lead compounds.

34 Furthermore, we demonstrated that sequence alignment, homology modeling and structural comparison may be useful for determining potential enzymes or receptors which can be eco-

friendly pesticide targets. 


\section{Introduction}

The traditional insecticides have made a major contribution to agriculture and health. But as a result of improper use and the inherent shortcomings of some insecticides, many showed negative impacts on the ecological environment. Therefore, eco-friendly insecticides have received global attention in recent years. But to predict and avoid potential ecological risk in the initial phase of insecticide discovery is a problem that has not been fully resolved to date.

The insect juvenile hormones (JHs) are methyl esters of farnesoic acid 10,11-epoxide (JH III) and related compounds, which function in the regulation of a number of insect physiological processes in insects including embryogenesis, larval and adult development, metamorphosis, reproduction, diapause, migration, polymorphism, and metabolism (Nijhout, 1994). JH biosynthesis proceeds in the corpora allata (CA) through the mevalonic acid (MVA) pathway, which insects share with most other organisms. By analogy with vertebrates, HMGCoA reductase (HMGR) has been postulated to be a key enzyme in the regulation of the MVA pathway in insects (Feyereisen, Pratt \& Hamnett, 1981). JH biosynthesis in insect CA is inhibited in vitro by compactin (Monger et al., 1982); mevinolin (Feyereisen \& Farnsworth, 1987; Couillaud, 1991); or fluvastatin (Debernard, Rossignol \& Couillaud, 1994). However, compactin shows poor inhibition of $\mathrm{JH}$ biosynthesis in vivo. Only repeated injections into Manduca sexta larvae induced the black pigmentation characteristic of $\mathrm{JH}$ deficiency; the black pigmentation is always followed by death within approximately 24 hours. In addition, compactin treatment by topical application has no effect on M. sexta larvae (Monger et al., 
59 1982). Fluvastatin injected into locusts inhibited JH biosynthesis in vivo, but by 12 hours, JH

60 biosynthesis had almost fully recovered, with no discernible effects on either JH-regulated

61 metamorphosis or oocyte maturation (Debernard, Rossignol \& Couilland, 1994). However,

62 the use of HMGR inhibitors for pest control has not been fully explored. In addition, because

63 HMGR is an enzyme which exists in most organisms, its status as an eco-friendly insecticide

64 target remains unclear.

65 In the present study, we predict the possibility of HMGR as an eco-friendly insecticide

66 target by using sequence alignment, homology modeling, and structural comparison. The

67 effects of three commercial HMGR inhibitors on JH biosynthesis was assayed by using $M$.

68 sexta, Apis mellifera, and Diploptera punctata as experimental animals in vitro to validate our

69 predictions. Finally, the possible applicability of these compounds for pest control was

70 demonstrated in this paper.

\section{Materials and methods}

\subsection{Insects}

Larvae of the tobacco hornworm, M. sexta, were raised from eggs provided by Carolina Biological Supply Company (Burlington, NC, USA) and reared on an artificial diet (Bio-Serv, $\mathrm{NJ}, \mathrm{USA})$ at $25^{\circ} \mathrm{C}$ under a long-day (16 h light/8 h dark) photoperiod (Bell \& Joachim, 1976).

Pharate $5^{\text {th }}$ instar larvae were set aside $4-7 \mathrm{~h}$ before lights off. The larvae molted within a few hours and were designated day 0 . At the start of wandering, indicated by the appearance of a prominent dorsal vessel, the larvae were transferred to plastic vials containing vermiculite until 
79

80

81

82

83

pupation. Freshly ecdysed pupae were transferred to a chamber containing a tobacco plant and $10 \%$ sucrose under a long-day photoperiod into which the adult moths emerged (Lee,

\section{Chamberlin \& Horodyski, 2002).}

Newly emerged mated female D. punctata (day 0) were isolated from stock cultures.

Mating was confirmed by the presence of a spermatophore. Stocks and isolated females were fed Lab Chow and water ad libitum, and were kept at $27 \pm 1^{\circ} \mathrm{C}$ and $50 \pm 5 \%$ relative humidity with a 12 h light/12 h dark cycle (Kai et al., 2009). in an incubator at $34^{\circ} \mathrm{C}$ and $80 \%$ relative humidity, fed a diet that was prepared with $40 \%$ pollen collected from combs and $60 \%$ honey. Fourth instar worker larvae were distinguished by the differences in maximum width of their head capsules (Rachinsky, Tobe \& Feldlaufer, 2000).

\subsection{Chemicals}

$L\left[{ }^{14} \mathrm{C}-\mathrm{S}\right.$-methyl $]$ methionine was purchased from Amersham Biosciences (Piscataway, $\mathrm{NJ}$, USA). HMGR inhibitors fluvastatin, pitavastatin and lovastatin and high-performance liquid chromatography (HPLC)-grade isooctane was purchased from Sigma-Aldrich (St. Louis, MO, USA).

\subsection{Bioassays}

\subsubsection{Assays for JH biosynthesis assays in vitro}

Rates of JH biosynthesis were determined in vitro by using the modified radiochemical 
assay (Tobe \& Clarke, 1985; Tobe \& Pratt, 1974). The radiochemical assays for JH biosynthesis were performed with CA from unfed day 1 fifth instars of $M$. sexta, day 7 adult female $D$. punctata and fourth instar workers of A. mellifera, respectively. HMGR inhibitors were dissolved in medium 199 (GIBCO) for assay as described previously (Lee, Chamberlin \& Horodyski, 2002; Kai et al., 2009) and used on the same day that the inhibitors were prepared. Each pair of CA was incubated for $3 \mathrm{~h}$ at $30^{\circ} \mathrm{C}$ in $100 \mu \mathrm{L}$ of medium 199 with Hanks' salts, $L$ glutamine, $25 \mathrm{mM}$ HEPES buffer (pH 7.2), $1.3 \mathrm{mM} \mathrm{Ca}^{2+}$ and $2 \%$ Ficoll, containing $L\left[{ }^{14} \mathrm{C}-\mathrm{S}-\right.$ methyl] methionine (40 $\mu \mathrm{M}$, specific radioactivity $1.48-2.03 \mathrm{GBq} / \mathrm{mmol})$ in the dark with gentle shaking. After incubation, both medium and CA were extracted with isooctane. The isooctane phase was removed and its radioactivity determined by liquid scintillation spectrometry. Inhibition of JH biosynthesis was calculated as percent activity compared with the control group (i.e. no HMGR inhibitor added). The $\mathrm{IC}_{50}$ values for the test compounds were calculated by using GraphPad Prism version 5.0.

\subsubsection{Assays for JH biosynthesis in vivo}

\subsubsection{Injection}

Injections of HMGR inhibitors ( $2 \mu \mathrm{L}$ volume, and $1 \mu \mathrm{M}$ concentration) in newly molted fifth instar $M . \operatorname{sexta}$ (day 0 ) were carried out using a $10 \mu \mathrm{L}$ Hamilton-syringe. The final concentrations of the injected inhibitor in the hemolymph were approximately $4 \mathrm{nM}$. Control larvae were similarly injected, but with $2 \mu \mathrm{L}$ of double distilled water. Larvae were first anesthetized by cooling on ice and then injected between the seventh and eighth spiracles near 
118 the horn, close to the posterior heart chamber. These animals were assayed for JH biosynthesis at

119 day 1 using the method described in Section 2.3.1. Each group of inhibitor-injected animals was

120

121

122

123

124

125

126

127

128

129

130

131

132

133

134

136

compared with a group of water-injected animals treated concurrently.

\subsubsection{Topical Application}

Solutions of HMGR inhibitors $(5 \mu \mathrm{L})$ were applied to the dorsal abdomen of $M$. sexta fifth instars at day 0 , and animals were assayed for JH biosynthesis at day 3 as described (see Section 2.3.1). The concentration of the inhibitors (in $20 \%$ DMSO and $80 \%$ acetone) used in the bioassays was $100 \mu \mathrm{M}$. Each larva received $0.5 \mathrm{nmol}$ inhibitor in the topical cuticular assays. Controls were treated with the solvent.

\subsubsection{Oral administration}

Newly molted fifth instars of $M$. sexta were immediately fed with $5 \mu \mathrm{L}$ inhibitor solution (1 $\mu \mathrm{M}$ concentration); subsequently, these animals were fed on the normal diet. Control larvae were similarly fed, but with $5 \mu \mathrm{L}$ of double distilled water. JH biosynthesis in these treated animals was assayed one day 1ater by using the radiochemical assay.

\subsubsection{Assays for ovicidal activity on M. sexta}

M. sexta eggs that had been deposited on a paper filter were briefly immersed in solutions of the HMGR inhibitor $\left(\mathrm{H}_{2} \mathrm{O}\right.$ containing $0.2 \%$ DMSO as co-solvent, concentrations ranged from $1 \mu \mathrm{M}$ to $1000 \mu \mathrm{M})$. After the test solution had dried, eggs were maintained in Petri dishes. Five days later, the mortality (numbers of eggs that failed to hatch) was determined, relative to 
137 untreated controls. (No eggs hatched after five days in either the treatment or control groups.

138

139

140

141

142

143

144

145

146

147

148

149

150

151

152

153

154

155

\subsubsection{Assays for impact of feeding on M. sexta larval growth and mortality}

Three groups of larvae were used for feeding assays. Newly hatched or newly molted $M$. sexta larvae were fed with HMGR inhibitor solution $(2 \mu \mathrm{L}$ for first and second instars, $3 \mu \mathrm{L}$ for third instars, and $5 \mu \mathrm{L}$ for fourth and fifth instars) at the beginning of the stadium, and then reared on the normal artificial diet until the next ecdysis. Larval mortality and insect growth were recorded after treatment.

\subsubsection{Statistics}

Data presented as percentages were log-transformed before statistical analyses. Data were analysed by using a one-way analysis of variance (ANOVA) with Dunnett's multiple comparison test as the post hoc determination of significance by using GraphPad Prism version 5.0. Doseresponse curves were prepared with GraphPad Prism. Values are expressed as mean \pm standard errors (S.E.M.) with $N$ indicating the number of samples measured ( $N$ is $8-20)$.

\subsection{Sequence Alignment of HMGR}

A sequence database of all known HMGR was collected from the literature and GenBank by using a combination of BLAST and keyword searches. Amino acid multiple sequence alignments for HMGR were constructed with ClustalW (Thompson, Higgins \& Gibson, 1994) and adjusted by eye to ensure structural motifs were maintained. Poorly aligned regions and major gaps were deleted. 
156

157

158

159

160

161

162

163

164

165

166

167

\subsection{Homology modeling}

Because there was no crystal structure of insect HMGR, the homology models of HMGR of M. sexta, A. mellifera, and D. punctata were prepared respectively, to explore the threedimensional structural differences of the HMGR from different organisms, especially the differences at their active site. A crystal structure of human HMGR in complex with Fluvastatin (PDB ID: 1HWI) was used as the 3D coordinate template for the homology modeling (Istvan \& Deisenhofer, 2001). The homology models for HMGRs were generated by using the FUGUE and ORCHESTRAR modules in Sybyl. The initial model was optimized energetically by using the minimize program with steepest descent algorithm, AMBER7 FF99 as the force field and Gasteiger-Huckel as the atomic point charges. The minimization was terminated when the RMS gradient convergence criterion of $0.05 \mathrm{kcal} /(\mathrm{mol} \cdot \AA)$ was reached. The qualities of these models were analyzed by PROCHECK (Laskowski et al., 1993).

\subsection{Docking calculations}

constructed by using the 2D sketcher module in Sybyl. Minimum energy conformations of all structures were calculated with the Minimize module of Sybyl. The force field was MMFF94 with an $8 \AA$ cutoff for nonbonded interactions, and the atomic point charges were also calculated with MMFF94 (Halgren, 1999). Minimizations were achieved with the steepest descent method for the first 100 steps, followed by the Broyden-Fletcher-Goldfarb-Shanno (BFGS) method until the Root-Mean-Square (RMS) of the gradient became less than $0.005 \mathrm{kcal} /(\mathrm{mol} \cdot \AA)(\mathrm{Head}$ 
176

\& Zerner, 1985; Kai et al., 2006).

The Surflex-Dock (Spitzer \& Jain, 2012) module implemented in the Sybyl program was

used for the docking studies. The 3-D structures of M. sexta, A. mellifera and D. punctata were performed with homology modeling. Each inhibitor was docked into the binding site of the corresponding protein by an empirical scoring function and a patented search engine in SurflexDock applied with the automatic docking. Other parameters were established by default in the software.

\subsection{Molecular dynamics simulations}

Docking calculations as described above were performed for each inhibitor in complex with HMGR in which energy was minimized and used the minimized program in Sybyl-X 2.0 with the optimization algorithm BFGS (Head \& Zerner, 1985). The force field was AMBER7 FF99 and the atomic point charge was Gasteiger-Huckel for 500 steps to remove bad contacts (Kai et al., 2006). The system was equilibrated at $400 \mathrm{~K}$ for $0.1 \mathrm{~ns}$ followed by data collection, at regular intervals, for $10 \mathrm{~ns}$. Each structure collected was subjected to $0.1 \mathrm{~ns}$ of simulated annealing to $300 \mathrm{~K}$. The final 100 structures were energy-minimized and clustered using cutoff distance of $<0.2 \mathrm{~nm}$. AMBER7 FF99 was used for force field and Gasteiger-Huckel for charges in molecular dynamics simulation using the dynamics program of Sybyl.

\section{Results}

\subsection{Sequence analysis}


from different species were aligned relative to that of $M$. sexta, and the identity values were

recorded (Figure 1). The HMGR sequences of Blattaria, Isoptera, Coleoptera, Hymenoptera,

Homoptera and Diptera were significantly different from Lepidoptera, (Dunnett's multiple

comparison test of identity values) (Figure 1A). The identity values of more distantly related

200

organisms, i.e., Malacostraca, Actinopterygii, Amphibia, Aves, Mammalia, Monocotyledoneae,

and Dicotyledoneae were below 60\% (Figure 1B), in comparison with the identity value of the

Lepidoptera (approximately 90\%). This suggests that the HMGR sequences of Lepidopteran

insects are significantly different from other organisms.

\subsection{Homology modeling, docking and Molecular dynamics optimization}

With the goal of discovery of an eco-friendly insecticide target, the three-dimensional

structures (especially the active site structures) of different species were analyzed. The HMGR of

M. sexta, A. mellifera and D. punctata were selected for the structural comparison. The

homology models of M. sexta, A. mellifera and D. punctata were generated using the crystal

structure of $H$. sapiens (PDB ID: 1HWI) as the template. To select the best model, we checked

the structural validity by PROCHECK (http://services.mbi.ucla.edu/SAVES). The geometry of

the final refined models were evaluated with Ramachandran plot calculations computed using the

PROCHECK program. The torsion angles of $\varphi$ and $\psi$ (the two torsion angles of the

polypeptide chain, also called Ramachandran angles, describe the rotations of the polypeptide 
215 was represented in the Ramachandran plot as shown in Figure S1. The Ramachandran plot

216 showed $89.7 \%$ of the residues of $M$. sexta, $88.2 \%$ residues of $A$. mellifera and $90.5 \%$ residues of

217 D. punctata existed in the most favored regions. The percentages of residues in disallowed

218 regions of $M$. sexta, A. mellifera and D. punctata are $0.0 \%, 0.1 \%$ and $0.3 \%$, respectively. This

219 indicated that the backbone dihedral angles, phi and psi, of the three homology models were

220 reasonably accurate.

One HMGR structure was assembled with the same four subunits. Each two adjacent

subunits constituted a ligand binding pocket, which means one HMGR contained the same four

binding pockets. Lovastatin, a commercial HMGR inhibitor, was used to identify the binding

pocket of the aforementioned HMGR structures with docking calculations. Molecular dynamics

225

(MD) simulation of the three homology models complexed with lovastatin as the ligand were

226

performed for $10 \mathrm{~ns}$ to obtain the stable and low energy conformations. By reporting the root

227

mean square deviation (RMSD) of the protein structure from the starting model, the receptor

changes in structure and reaches a relatively stable conformational minimum after approximately

3 ns. The conformations with the lowest energy of the final 100 structures from the MD

simulation were selected as the final structures.

\subsection{Structure comparison}

Both subunits that constituted the binding pocket of $A$. mellifera and D. punctata formed

hydrogen bonds with lovastatin, whereas only one subunit of $M$. sexta can form hydrogen bonds with the ligand (Arg 579 and Lys 680 of chain A). This suggested that the binding pocket of $M$. 
235 sexta is more flexible.

236 The surface properties of the binding pocket of the above three structures were defined

237 using MOLCAD calculations (an interactive visualization of molecular scenarios) in Sybyl to

238 analyse these binding pockets. Figure 2 sketches the molecular surfaces of the pockets of these

239 four HMGRs. The cavity of $M$. sexta was smooth and did not penetrate deep into the structure

240 compared with the cavities of $A$. mellifera and D. punctata (Figure 2). This result was in

241 accordance with the results of hydrogen bond interaction. The electrostatic potential of the

242 binding pockets of $M$. sexta was positively-charged, because the whole surface of the pocket was

243 colored in yellowish green, whereas that of $A$. mellifera was electroneutral (Figure 2). The front

244 of the binding pocket of D. punctata was colored with blue, which suggests that some part of this

245 pocket is electronegative. The lipophilic potential of the binding pocket of M. sexta was

246 lipophilic, colored with brown, whereas that pocket of D. punctata was more hydrophilic,

247 colored with blue. The green color suggested that the pocket of A. mellifera is neutral (Figure 2).

248 These results show that the active pockets of the three species are different, suggesting that it is

249 possible to design eco-friendly insecticides using differences in surface properties. Thus, the

250 insect HMGR may represent a potential eco-friendly insecticide target.

251 The surface properties of these three pockets suggests that increasing the molecular volume,

252 electronegativity and lipophilicity of the ligand can strengthen the binding affinity between

253 ligand and HMGR of M. sexta, whereas it also can weaken the binding affinities with other

254 species. 

lepidopteran pest $M$. sexta, all the compounds have potent inhibitory activity on JH biosynthesis. inhibitors tested in the present work are safe for honeybees. Similarly, the inhibitory effects on D. punctata were much lower than on M. sexta. The above results suggest that insect HMGR (in particular, lepidopteran HMGR) might be an eco-friendly insecticide target. type II statins should be good lead compounds for new insecticide design. In addition to their effects in vitro, the statins also showed significant effects on $\mathrm{JH}$ production by $M$. sexta following treatment in vivo.

\subsubsection{Injection}


$2755.8 \%$, respectively.

276

277

278

279

280

281

282

283

284

285

286

287

\subsubsection{Effects on JH biosynthesis following oral administration}

In addition to the effects in the injection bioassays, the statins also showed a significant effect on $\mathrm{JH}$ production following oral administration (Figure $3 \mathrm{~A}$ ). In bioassays at $1 \mu \mathrm{M}$, inhibition of JH biosynthesis by fluvastatin, pitavastatin and lovastatin was $58.9 \pm 8.9 \%, 54.4 \pm$ $3.1 \%$ and $62.6 \pm 3.4 \%$, respectively. This suggests that HMGR inhibitors can also inhibit JH biosynthesis following oral administration.

\subsubsection{Topical application}

In topical cuticular assays, no compound demonstrated any effect on JH biosynthesis (Figure 3B); this might be attributable to the poor cuticular penetration of the reagents, and indicates that HMGR inhibitors are unlikely to be contact insecticides.

\subsection{Pest control application}

\subsubsection{Ovicidal effects}

The three compounds also demonstrated significant activity on viability of $M$. sexta eggs (Table 2). At a concentration $(100 \mu \mathrm{M})$, the mortality of eggs following treatment with fluvastatin, pitavastatin and lovastatin was $76.1 \%, 100 \%$ and $100 \%$, respectively. A concentration of $50 \mu \mathrm{M}$ of these compounds gave about $50 \%$ inhibition.

\subsubsection{Larvicidal effects following oral administration of statins}


The experiment in section 3.4.2 demonstrated that the statins have a significant effect on JH

294

295

296

297

298

299

300

301

302

303

304

305

306

307

308

309

310

311

312

biosynthesis following oral administration. Accordingly, a stomach toxicity test was performed.

We first determined which instars of $M$. sexta were most sensitive to the statins following

feeding at high concentration $(1000 \mu \mathrm{M})$ in $1^{\text {st }}, 2^{\text {nd }}$ and $3^{\text {rd }}$ instars, respectively, and recorded the

mortality in Table 3. All larvae that commenced feeding from the $1^{\text {st }}$ stadium died prior to

pupation, and most died in the $1^{\text {st }}$ stadium. Larvae fed from the $2^{\text {nd }}$ stadium were also sensitive to the statins with a high mortality (above $85 \%$ ). However, when the treatment commenced from the $3^{\text {rd }}$ stadium, the mortality before the next molt was less than $10 \%$, and mortality just prior to pupation was less than $40 \%$. For all the inhibitors, the earlier instars were more sensitive than the later instars.

We then topically treated larvae with different concentrations of the statins, commencing with $1^{\text {st }}$ instars, and recorded larval mortality (Table 4). The statins showed significant larvicidal activity at $100 \mu \mathrm{M}$. The $\mathrm{IC}_{50}$ values of fluvastatin, pitavastatin and lovastatin were $2101 \mu \mathrm{M}$, 63.0 $\mu \mathrm{M}$ and $298.3 \mu \mathrm{M}$, respectively.

In the dead larvae, the most striking characteristic was the darkening of the cuticle in some animals as well as molting disturbances (Figure 4). This is consistent with the phenomenon of the inhibition of JH biosynthesis (Monger et al., 1982).

\subsubsection{Growth regulation}

A long-term feeding study with low concentration of inhibitors was performed to identify their effects on growth. We fed larvae with inhibitors starting with $1^{\text {st }}$ instars, and recorded the 
313 number of days from hatching to the $5^{\text {th }}$ stadium (larvae which died before the $5^{\text {th }}$ stadium were

314 not recorded). For fluvastatin, the number of days from newly hatched larvae to the $5^{\text {th }}$ stadium

315 at $100 \mu \mathrm{M}, 10 \mu \mathrm{M}$ and $1 \mu \mathrm{M}$ was $17.3,13.6$ and 13.5 days, respectively. In the control group

316 (not fed inhibitors), the interval was 11.9 days. It appears that the HMGR inhibitors significantly

317 slowed the growth rate of M. sexta. Figure 5A shows the difference in growth more clearly.

318 Three larval groups hatched on the same day; one was fed normal food as control, one was

319 treated with $100 \mu \mathrm{M}$ of fluvastatin, and the other was treated with $1 \mu \mathrm{M}$ of fluvastatin. The

320 difference in size was readily apparent by comparison with the control $4^{\text {th }}$ instars. The other

321 statins also showed the same growth effect as fluvastatin (Figure S2).

322 Following treatment with a low concentration $(1 \mu \mathrm{M})$ of the statins, we observed that pupation of these larvae was not normal. Most larvae died in the process of pupation as a result of malformation (Figure 5B). This suggests that the statins can be potent insect growth regulators.

\section{Discussion} to combat growing problems associated with resistance, environmental pollution, accumulation of pesticide residues in the food chain and detrimental effects on non-target organisms. Hence, the need for eco-friendly insecticides with safe and novel modes of action or targets is becoming increasingly important. In this study, we have not only focused on elucidating new eco-friendly insecticide targets and lead compounds, but also attempted to provide an empirical method for eco-friendly insecticide discovery. 
Insect JHs are a group of structurally related sesquiterpenoids that regulate a number of

physiological processes including embryogenesis, larval and adult development, metamorphosis,

335

336

337

338

339

340

341

342

reproduction, pheromone biosynthesis, diapause, migration, polymorphism, and metabolism

(Nijhout, 1994; Kerkut \& Gilbert, 1985; Gilbert, Granger \& Roe, 2000). To our knowledge, the

occurrence of JHs and related sesquiterpenoids such as methyl farnesoate is confined to animals

in the Arthropoda. It has been demonstrated that the design of $\mathrm{JH}$ mimics or anti-JH agents is an

effective strategy for insecticide discovery. Screening new targets involved in JH biosynthesis

has been a subject of study for two decades (Bede et al., 2001). As HMGR has been postulated to

be a key enzyme in the regulation of the MVA pathway in insects, some HMGR inhibitors

(statins) have been used to investigate their effects on $\mathrm{JH}$ biosynthesis. Compactin, mevinolin

and fluvastatin have been demonstrated to be potent inhibitors of JH biosynthesis in vitro,

whereas studies of their effects in vivo are incomplete (Monger et al., 1982; Couillaud, 1991).

Thus, to date, HMGR has not been used as a potential insecticide target. Furthermore, whether

HMGR inhibitors have detrimental effects on non-target organisms remains unknown.

We predicted and evaluated the ecological safety of HMGR by using sequence alignment

and structural comparison. Sequence analysis showed that the Lepidoptera differ from other

organisms. Zapata et al. tested the effects of two HMGR inhibitors, fluvastatin and compactin, on

HMGR activity of Blattella germanica. Both compounds significantly inhibited the enzymatic

activity at a high concentration (50 $\mu \mathrm{g}$ per animal) by approximately $25 \%$ in vivo (Zapata et al.,

2002). The inhibition by fluvastatin on $M$. sexta HMGR was approximately $60 \%$ at a low 
353

354

355

356

357

concentration $(0.8 \mathrm{ng}$ per animal in an injection assay and $2 \mathrm{ng}$ per animal following oral administration, respectively) in our study. BLAST showed that the identity value between $B$. germanica HMGR and M. sexta HMGR was 47\%. Bacterial HMGR has a low sequence identity value compared with M. sexta HMGR. Lovastatin inhibited Pseudomonas mevalonii at a high concentration $\left(K_{i}\right.$ value $\left.=0.53 \mathrm{mM}\right)($ Hedl \& Rodwell, 2004). However, the inhibitory effect of lovastatin on $M$. sexta was much greater than on $P$. mevalonii $\left(\mathrm{IC}_{50}\right.$ value $\left.99 \mathrm{nM}\right)$. The identity value between $P$. mevalonii HMGR and $M$. sexta HMGR was 23\%. In our experiment, the identity value of $A$. mellifera and $D$. punctata versus $M$. sexta was $48 \%$ and $50 \%$. Three HMGR inhibitors have no or little effect on A. mellifera and D. punctata; however these compounds are potent inhibitors in $M$. sexta. This suggests that there might be a link between the sequence alignment data and inhibition.

The sequence and 3-D structure (in particular, the molecular potential surface properties) of lepidopteran HMGR differs from other organisms (A. mellifera and D. punctata) in this study. Assays of JH biosynthesis in the presence of HMGR inhibitors in different insect species showed that those inhibitors have potent effects on the lepidopteran pest $M$. sexta, but are much less effective on A. mellifera (Hymenoptera) and D. punctata (Blattodea). This confirms our suggestion regarding the value and assessment of the ecological safety of HMGR as an insecticide target candidate.

The applicability to pest control is crucial in the evaluation of the potential of a chemical to act as an insecticide. Previous studies indicated that HMGR was the control point in JH 
373

374

biosynthesis in M. sexta (Monger et al., 1982). As a consequence of limited experimental data on the application of HMGR inhibitors for pest control, we tested the effects in vivo of three HMGR inhibitors on M. sexta. Our present study revealed that HMGR inhibitors can be potential insecticide candidates with excellent ovicidal activity, larvicidal activity and growth regulatory effects. In the fat body, HMGR was crucial to vitellogenesis and reproduction. Short-term assays showed that HMGR inhibitors reduce the protein levels and enzymatic activity of HMGR, and long-term experiments revealed that fluvastatin impairs embryo development (Zapata et al., 2002). Our work clearly indicates that HMGR is a key enzyme in embryogenesis, larval and adult development and metamorphosis. In Agrotis ipsilon, fluvastatin also disrupted normal spermatophore transfer (Duportets et al., 1998). It suggested that insect HMGR can be an insecticide target and its inhibitors could be insecticide lead compounds.

We conclude that an empirical method of discovery of eco-friendly insecticides encompasses the prediction of ecological safety of insecticide target candidates and the probability of the application for pest control. The steps for ecological safety prediction are as follows:

(1) Collect sequence data of insecticide target candidates from all species of interest.

(2) Perform sequence alignment of each species and compare to the selected target pest. Statistically analyze the identity values from sequence alignments. If there is no difference between pest and non-target organisms, this candidate is a likely to be an ecotoxic insecticide target. If not, go to the next step. 
(3) Perform structural comparisons and docking studies with ligands of pests and other nontarget organisms. If there is no difference between their structures (especially the binding pockets) or binding affinities, this candidate is a possible eco-toxic insecticide target. If not, it could be an eco-friendly insecticide target. New eco-friendly insecticides can be designed based on structural differences.

Although this method of prediction cannot replace the requisite toxicity tests, it can avoid unnecessary waste, save manpower, material and time in the discovery of new ecofriendly insecticides.

\section{Conclusion}

We have demonstrated that insect HMGR can be a potential selective insecticide target, and its inhibitors can be potential selective insecticides. Our research should be helpful for designing new selective insecticides. Furthermore, we have demonstrated that sequence alignment, homology modeling and structural comparison can be used to determine which enzymes or receptors could be selective pesticide targets. Pest control applications have shown that the HMGR inhibitors are potential insect growth regulators, especially for lepidopteran pest control. 
409 Acknowledgments

410 The authors thank Dr. Frank M. Horodyski (Ohio University, Athens, OH) for providing

411 bioassay equipment.

412

413

414

415

416

417

418

419

420

421

422

423

424

425

Peer] reviewing PDF | (2016:08:12890:2:0:NEW 29 Nov 2016) 
428

430

431

432

433

434

435

436

437

438

439

440

441

442

443

444

445

446

447

448

\section{References}

Bede JC, Teal PE, Goodman WG, Tobe SS. 2001. Biosynthetic pathway of insect juvenile hormone III in cell suspension cultures of the sedge Cyperus iria. Plant physiology 127: 584-593

\section{DOI 10.1104/pp.010264.}

Bell RA, Joachim FG. 1976. Techniques for rearing laboratory colonies of tobacco hornworms and pink bollworms. Annals of the Entomological Society of America 69: 365-373 DOI http://dx.doi.org/10.1093/aesa/69.2.365.

Couillaud F. 1991. Evidence for regulation of juvenile hormone biosynthesis operating before mevalonate in locust corpora allata. Molecular and cellular endocrinology 77: 159-166 DOI

\subsection{6/0303-7207(91)90070-9.}

Debernard S, Rossignol F, Couillaud F. 1994. The HMG-CoA reductase inhibitor fluvastatin inhibits insect juvenile hormone biosynthesis. General and comparative endocrinology 95: 92-98

\section{DOI 10.1006/gcen.1994.1105.}

Duportets L, Dufour MC, Couillaud F, Gadenne C. 1998. Biosynthetic activity of corpora allata, growth of sex accessory glands and mating in the male moth Agrotis ipsilon (Hufnagel). Journal of Experimental Biology, 201: 2425-2432.

Feyereisen R, Farnsworth DE. 1987. Precursor supply for insect juvenile hormone III biosynthesis in a cockroach. Journal of Biological Chemistry 262: 2676-2681

Feyereisen R, Pratt GE, Hamnett AF. 1981. Enzymatic synthesis of juvenile hormone in locust corpora allata: Evidence for a microsomal cytochrome P-450 linked methyl farnesoate epoxidase. European Journal of Biochemistry 118: 231-238 DOI 10.1111/j.1432- 
449

450

451

452

453

454

455

456

457

458

459

460

461

462

463

464

465

466

467

468

469

1033.1981.tb06391.x.

Gilbert LI, Granger NA, Roe RM. 2000. The juvenile hormones: historical facts and speculations on future research directions. Insect biochemistry and molecular biology 30: 617644 DOI 10.1016/S0965-1748(00)00034-5.

Halgren, TA. 1999. MMFF VI. MMFF94s option for energy minimization studies. Journal of Computational Chemistry 20: 720-729 DOI 10.1002/(SICI)1096-987X(199905)20:7<720::AID-

$\mathrm{JCC} 7>3.0 . \mathrm{CO} ; 2-\mathrm{X}$

Head JD, Zerner MC. 1985. A Broyden-Fletcher-Goldfarb-Shanno optimization procedure for molecular geometries. Chemical physics letters 122: 264-270 DOI 10.1016/00092614(85)80574-1.

Hedl M, Rodwell VW. 2004. Inhibition of the Class II HMG-CoA reductase of Pseudomonas mevalonii. Protein Science 13:1693 - 1697 DOI 10.1110/ps.03597504.

Istvan ES, Deisenhofer J. 2001. Structural mechanism for statin inhibition of HMG-CoA reductase. Science 292: 1160-1164 DOI 10.1126/science.1059344.

Kai ZP, Huang J, Tobe SS, Yang XL. 2009. A potential insect growth regulator: synthesis and bioactivity of an allatostatin mimic. Peptides 30: 1249-1253 DOI 10.1016/j.peptides.2009.03.010. Kai ZP, Ling Y, Liu WJ, Zhao F, Yang XL. 2006. The study of solution conformation of allatostatins by 2-D NMR and molecular modeling. Biochimica et Biophysica Acta (BBA)Proteins and Proteomics 1764: 70-75 DOI 10.1016/j.bbapap.2005.10.010.

Kerkut GA, Gilbert CI. 1985. Comprehensive Insect Physiology, Biochemistry and Pharmacology. New York : Pergamon Press. 
Laskowski RA, MacArthur MW, Moss DS, Thornton JM. 1993. PROCHECK: a program to

check the stereochemical quality of protein structures. Journal of applied crystallography 26: 283-291 DOI 10.1107/S0021889892009944.

Lee KY, Chamberlin ME, Horodyski FM. 2002. Biological activity of Manduca sexta

allatotropin-like peptides, predicted products of tissue-specific and developmentally regulated alternatively spliced mRNAs. Peptides 23: 1933-1941 DOI 10.1016/S0196-9781(02)00181-X.

Monger DJ, Lim WA, Kézdy FJ, Law JH. 1982. Compactin inhibits insect HMG-CoA reductase and juvenile hormone biosynthesis. Biochemical and biophysical research communications 105: 1374-1380 DOI 10.1016/0006-291X(82)90939-1.

Nijhout HF. 1994. Insect Hormones. Princeton: Princeton University Press.

Rachinsky A, Tobe SS, Feldlaufer MF. 2000. Terminal steps in JH biosynthesis in the honey bee (Apis mellifera L.): developmental changes in sensitivity to $\mathrm{JH}$ precursor and allatotropin. Insect biochemistry and molecular biology 30: 729-737 DOI 10.1016/S0965-1748(00)00044-8.

Spitzer R, Jain AN. 2012. Surflex-Dock: Docking benchmarks and real-world application. Journal of computer-aided molecular design 26: 687-699 DOI 10.1007/s10822-011-9533-y.

Thompson JD, Higgins DG, Gibson TJ. 1994. CLUSTAL W: improving the sensitivity of progressive multiple sequence alignment through sequence weighting, position-specific gap penalties and weight matrix choice. Nucleic acids research 22: 4673-4680 DOI 10.1093/nar/22.22.4673.

Tobe SS, Clarke N. 1985. The effect of L-methionine concentration on juvenile hormone 
492 Tobe SS, Pratt GE. 1974. The influence of substrate concentrations on the rate of insect

493 juvenile hormone biosynthesis by corpora allata of the desert locust in vitro. Biochemical

494 Journal 144: 107-113 DOI 10.1042/bj1440107.

495 Zapata R, Martín D, Piulachs MD, Bellés X. 2002. Effects of hypocholesterolaemic agents on

496 the expression and activity of 3-hydroxy-3-methylglutaryl-CoA reductase in the fat body of the

497 German cockroach. Archives of Insect Biochemistry and Physiology 49:177-186 DOI

498 10.1002/arch.10018.

499 

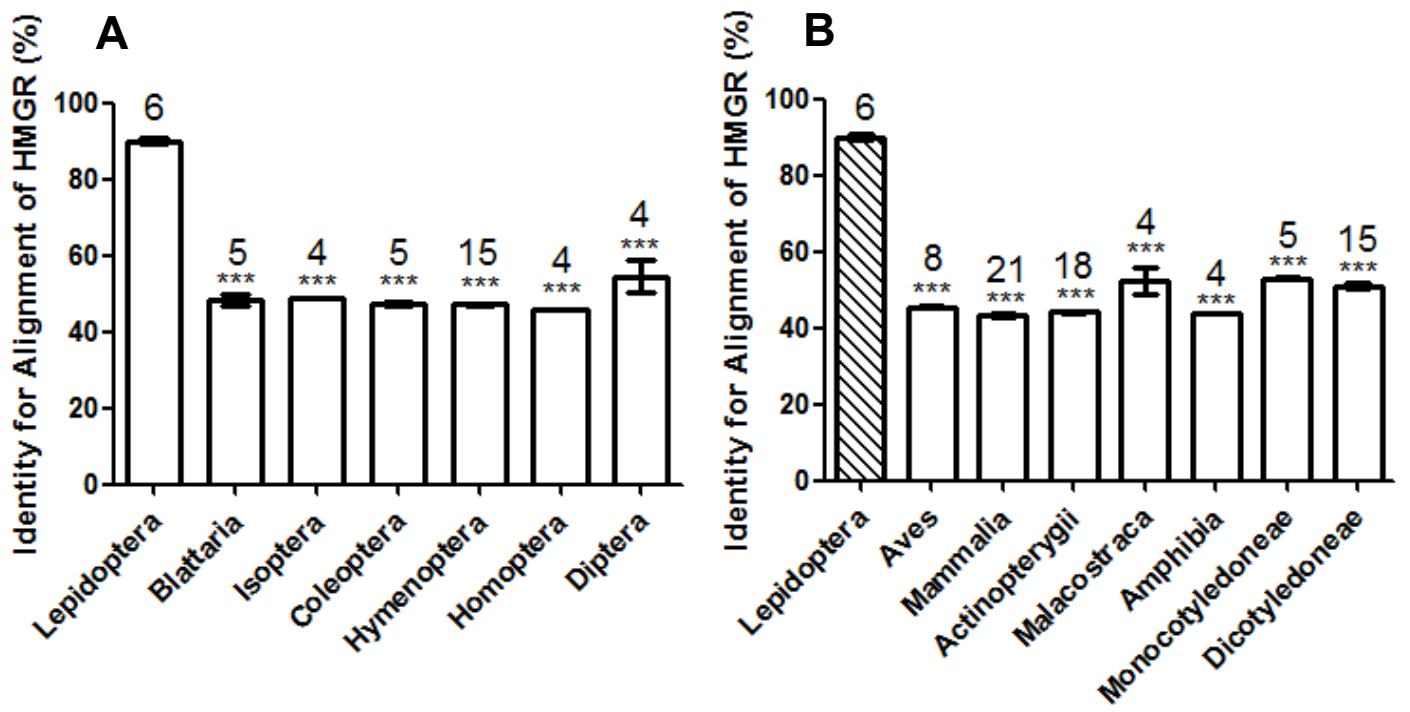

500

Figure 1. Comparison of the identity value of per species HMGR sequence relative to

Manduca sexta. (A) Asterisks indicate significant differences between Lepidoptera and other orders of Insecta as determined by Dunnett's multiple comparison test following one-way ANOVA: $* * *, P<0.001$. (B) Asterisks indicate significant differences between Lepidoptera and other organisms other than Insecta as determined by Dunnett's multiple comparison test following one-way ANOVA: ***, $P<0.001$. 


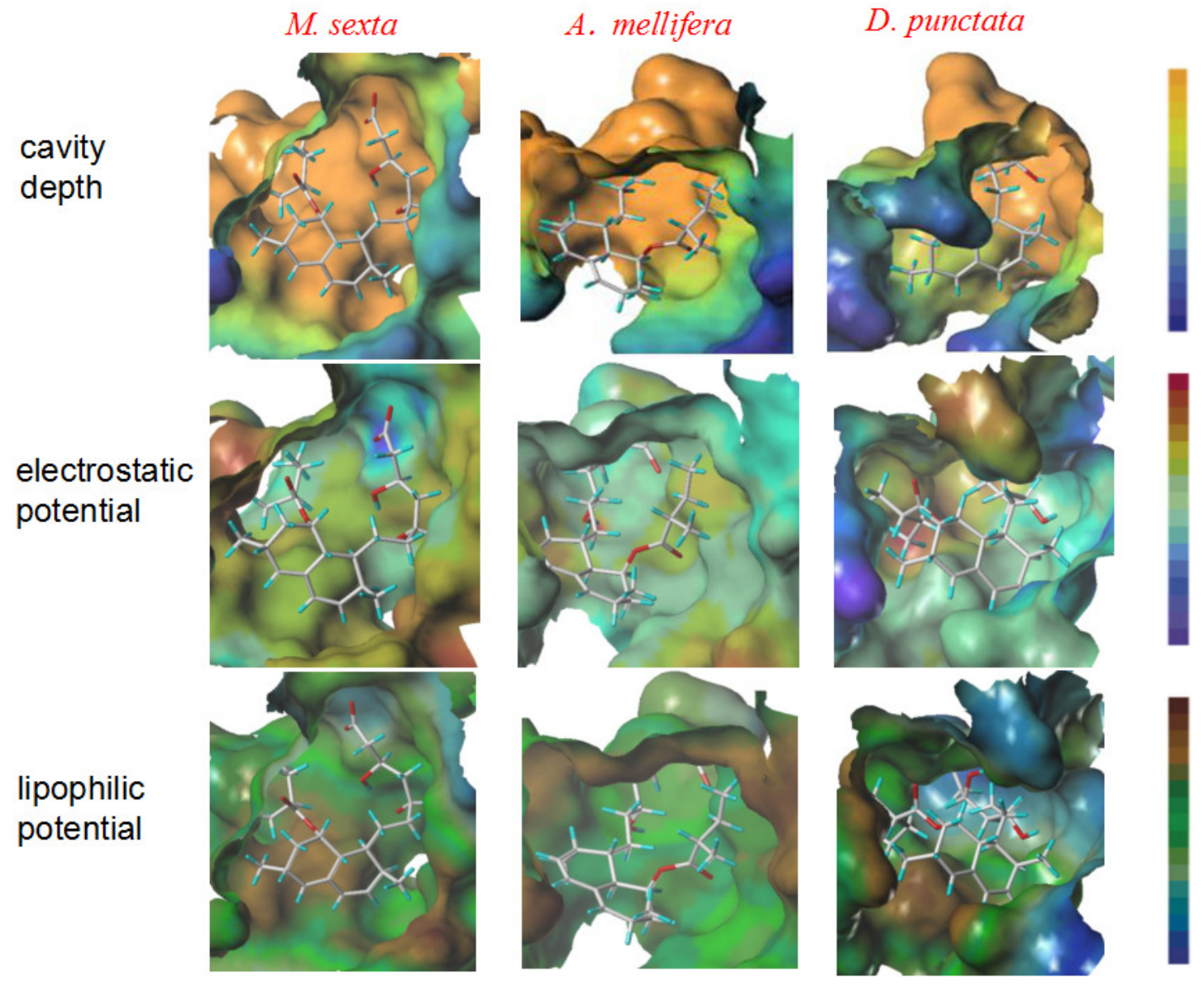

Figure 2. Structure comparison of binding pockets of M. sexta, A. mellifera and D. punctata

HMGR. The molecule in the pocket is lovastatin. In the presentation of cavity depth, the deep

blue colour represents the outermost surface of the structure, whereas the orange colour

511 represents the deepest part of the cavity. In the presentation of molecular electrostatic potential,

512 the deep blue colour represents the most negative potential, whereas the deep red colour

513 represents the most positive potential. In the presentation of the molecular lipophilic potential,

514 the deep blue colour represents the most hydrophilic parts of the surface, whereas the deep

515 brown colour represents the most lipophilic parts of the surface. 

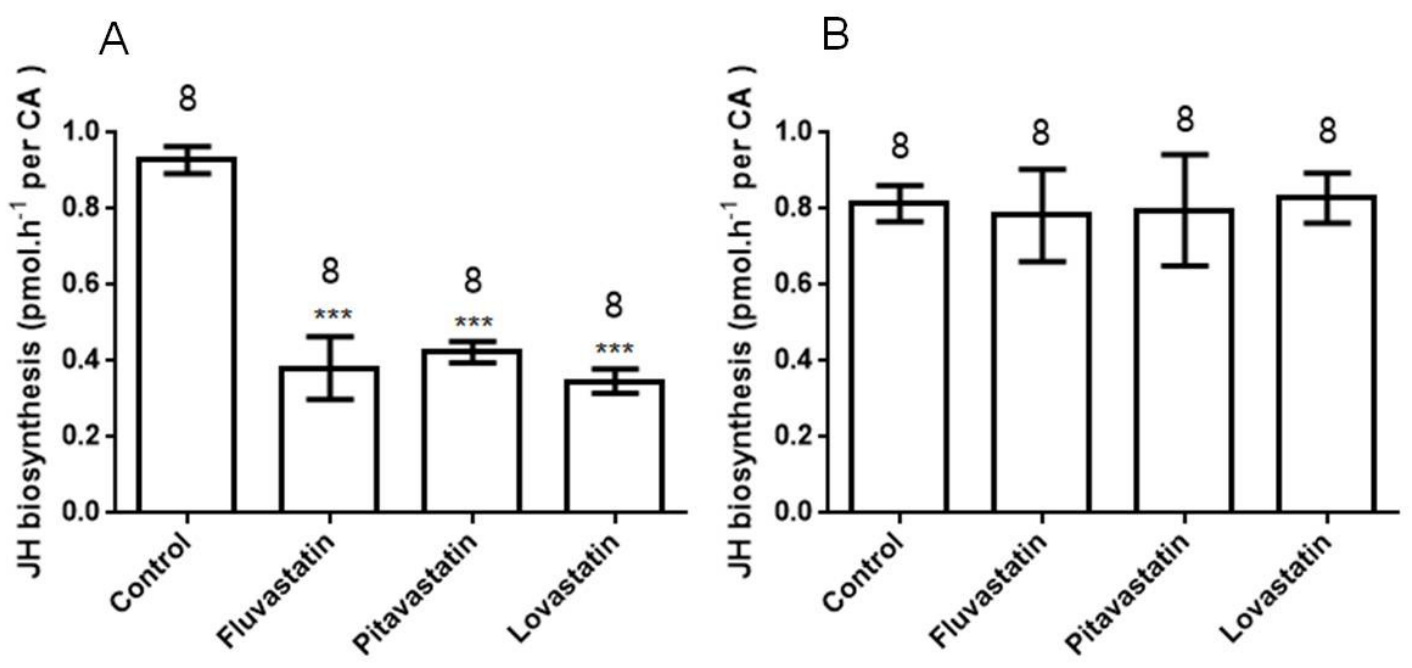

Figure 3. JH biosynthesis following oral treatment of various inhibitors (statins) (A) and topical

519 cuticular application of the same inhibitors (B). Each bar represents the mean \pm SEM. Asterisks indicate significant differences between inhibitor- and water-fed groups of animals as determined by Dunnett's multiple comparison test following one-way ANOVA: ***, $P<0.001$. 


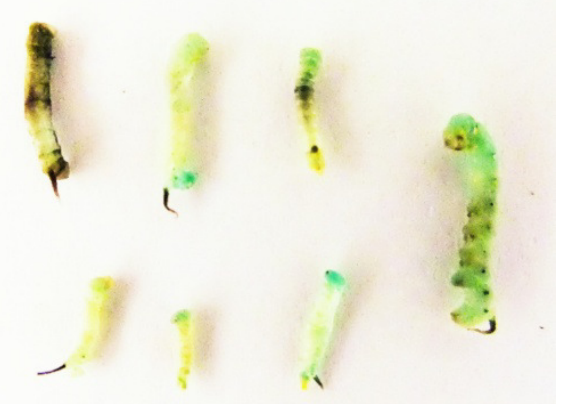

524 Figure 4. Dead larvae following feeding with $1000 \mu \mathrm{M}$ of fluvastatin as $1^{\text {st }}$ instars. Twenty 525 animals were used in this treatment. 
A

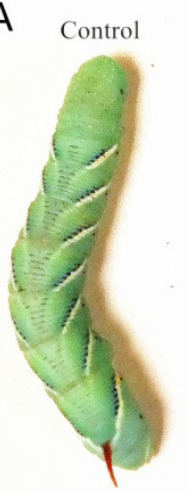

$1 \mu \mathrm{M}$

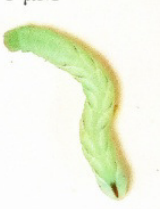

B

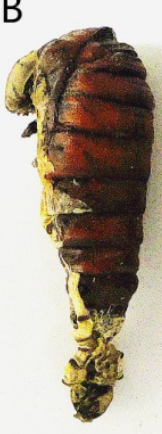

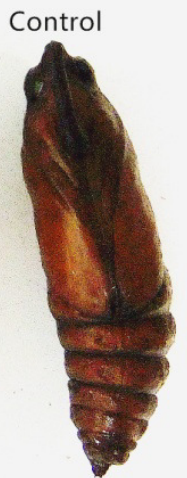

528

Figure 5. Developmental arrest and growth retardation in $M$. sexta following fluvastatin

treatment. There were twenty animals for each group. (A) Three larval groups hatched on the same day; one was fed normal food as the control; the others were treated with $1 \mu \mathrm{M}$ and 100 $\mu \mathrm{M}$ of fluvastatin, respectively. The difference in size is readily apparent in the control $4^{\text {th }}$ instars

532 (10 days after feeding). (B) Newly hatched larvae were treated with $1 \mu \mathrm{M}$ fluvastatin, most died 533 in the process of pupation as a result of malformation. 
535 Table 1. The $\mathrm{IC}_{50}$ values of HMGR inhibition of JH biosynthesis in vitro.

\begin{tabular}{|c|c|c|c|c|}
\hline Compound & Structure & $\begin{array}{l}\text { M. sexta, } \mathrm{IC}_{50} \\
\text { value }(\mathrm{nM})\end{array}$ & $\begin{array}{l}\text { A. mellifera, } \mathrm{IC}_{50} \\
\text { value }(\mathrm{nM})\end{array}$ & $\begin{array}{l}\text { D. punctata, } \mathrm{IC}_{50} \\
\text { value }(\mathrm{nM})\end{array}$ \\
\hline Fluvastatin & & 5.11 & 18100 & 150.0 \\
\hline Lovastatin & & 99.45 & No effect & 884.7 \\
\hline Pitavastatin & & 5.23 & 157500 & 395.2 \\
\hline
\end{tabular}

536 
538 Table 2. Ovicidal effect of HMGR inhibitors on $M$. sexta eggs at different concentrations.

539

\begin{tabular}{llll}
\hline Compound & $\begin{array}{l}\text { Concentration } \\
(\mu \mathrm{M})\end{array}$ & Number of eggs & Mortality (\%) \\
\hline Fluvastatin & 1000 & 60 & 100 \\
& 100 & 110 & 76.1 \\
& 10 & 86 & 11.9 \\
& 1 & 107 & 9.5 \\
Pitavastatin & 2000 & 69 & 100 \\
& 200 & 90 & 100 \\
& 20 & 90 & 40.3 \\
& 2 & 70 & 38.3 \\
Lovastatin & 1000 & 66 & 100 \\
& 100 & 99 & 99.99 \\
& 10 & 96 & 30.9 \\
Control & 0 & 88 & 37.8 \\
\hline
\end{tabular}

540 
542 Table 3. Mortality following feeding with statins at $1000 \mu \mathrm{M}$ during the first three stadia.

\begin{tabular}{|c|c|c|c|}
\hline Compound & Feeding treatment & Mortality before next molt (\%) & $\begin{array}{l}\text { Mortality before pupation } \\
(\%)\end{array}$ \\
\hline \multirow[t]{3}{*}{ Fluvastatin } & From $1^{\text {st }}$ instar & 100 & 100 \\
\hline & From $2^{\text {nd }}$ instar & 51 & 92 \\
\hline & From $3^{\text {rd }}$ instar & 6 & 13 \\
\hline \multirow[t]{3}{*}{ Pitavastatin } & From $1^{\text {st }}$ instar & 82 & 100 \\
\hline & From $2^{\text {nd }}$ instar & 80 & 85 \\
\hline & From $3^{\text {rd }}$ instar & 10 & 40 \\
\hline \multirow[t]{3}{*}{ Lovastatin } & From $1^{\text {st }}$ instar & 100 & 100 \\
\hline & From $2^{\text {nd }}$ instar & 90 & 100 \\
\hline & From $3^{\text {rd }}$ instar & 20 & 90 \\
\hline
\end{tabular}

543 
545 Table 4. Larval mortality following treatment of $1^{\text {st }}$ instars

\begin{tabular}{llll}
\hline Compound & Concentration $(\mu \mathrm{M})$ & Mortality before $3^{\text {rd }}$ instar $(\%)$ & Larval mortality $(\%)$ \\
\hline Fluvastatin & 1000 & 100 & 100 \\
& 100 & 25 & 25 \\
& 10 & 8.3 & 8.3 \\
& 1 & 16.7 & 16.7 \\
Pitavastatin & 1000 & 100 & 100 \\
& 100 & 100 & 100 \\
& 10 & 41.7 & 41.7 \\
Lovastatin & 1000 & 25 & 41.7 \\
& 100 & 100 & 100 \\
& 10 & 33.3 & 33.3 \\
& 1 & 0 & 8.3 \\
\hline
\end{tabular}

546 